\title{
Evaluation of silk fibroin electrogel coating for zirconia material surface
}

\author{
Yinying QU ${ }^{1,2}$, Guang HONG ${ }^{3,4}$, Lin LIU1 ${ }^{1}$, Keiichi SASAKI ${ }^{2}$ and Xiaodong CHEN 5 \\ ${ }^{1}$ Department of Orthodontics, Dalian Stomatological Hospital, 935 Changjiang Road, Shahekou District, Dalian 116027, China \\ ${ }^{2}$ Division of Advanced Prosthetic Dentistry, Graduate School of Dentistry, Tohoku University, 4-1 Seiryo-machi, Aoba-ku, Sendai 980-8575, Japan \\ ${ }^{3}$ Liaison Center for Innovative Dentistry, Graduate School of Dentistry, Tohoku University, 4-1 Seiryo-machi, Aoba-ku, Sendai 980-8575, Japan \\ ${ }^{4}$ Faculty of Dental Medicine, Airlangga University, JM. Prof. Dr. Moestopo 47, Surabaya 60132, Indonesia \\ ${ }^{5}$ Department of Prosthetics, Dalian Stomatological Hospital, 935 Changjiang Road, Shahekou District, Dalian 116027, China \\ Corresponding author, Guang HONG; E-mail: hong@m.tohoku.ac.jp
}

Zirconia is commonly used in dental applications. It has been reported that surface-modified zirconia implants showed better performance in vivo than machined zirconia implants. Silk fibroin electrogel is a good candidate for controlled drug delivery; however, the use of silk fibroin electrogel on zirconia implants has not previously been reported. The aim of this study was to investigate a method to coat zirconia implants with silk fibroin electrogel and evaluate the mechanical and biological properties of the coating. The results show that the wettability of the coating was close to that of sand-blasted and acid-etched (SLA)-treated zirconia, and the bond strength was larger than that of the coating prepared from silk fibroin aqueous solution. ATR-FTIR spectra provided evidence that the secondary structure changed during the electrogelation process. Culturing cells on the coating revealed its nontoxicity to osteoblast-like cells. Thus, it can be suggested that a silk fibroin electrogel coating is a promising biocompatible and degradable drugdelivery material for zirconia implants.

Keywords: Zirconia, Silk fibroin, Electrogel coating, Dental implant surface modification

\section{INTRODUCTION}

Titanium is a traditional material for manufacturing implants widely used in clinics, and has been applied in many biomedical engineering applications, such as total hip arthroplasty and surgical management of bone fracture ${ }^{1)}$. In the dental field, titanium material is used for implants, temporary anchorage devices, and frameworks for bone regeneration ${ }^{2,3}$. In some studies, the accumulation of titanium particles was detected in tissues close to the implants and local lymph nodes ${ }^{4,5}$. The potential local and systematic toxic effects, as well as the gray color of titanium, have resulted in controversy over its use as an implant material.

Since the 1980s, zirconia has been used extensively in medical and dental fields based on its excellent biocompatibility, ivory color, and favorable mechanical properties ${ }^{6,7)}$. Therefore, zirconia is one of the most promising implant materials. However, at low temperatures and in humid environments, zirconia undergoes a phase transition, leading to material degradation and a reduction in its mechanical strength. To solve this problem, yttria-stabilized zirconia was applied ${ }^{8,9)}$. To further enhance the mechanical performance of yttria-stabilized tetragonal zirconia polycrystals (Y-TZP), a Ce-TZP-based nanostructured zirconia/alumina composite (NANOZR) was developed ${ }^{10)}$. NANOZR has a high fracture strength and fracture toughness, as well as good biocompatibility ${ }^{11,12}$. When we use the NANOZR as a dental implant material,

Color figures can be viewed in the online issue, which is available at J-STAGE.

Received Jul 17, 2018: Accepted Dec 18, 2018

doi:10.4012/dmj.2018-228 JOI JST.JSTAGE/dmj/2018-228 biocompatibility plays a critical role in the generation and maintenance of bone tissue around the implants, which is referred to as osseointegration. Surface properties are an important factor for improving the biocompatibility of NANOZRs. There are also several methods to modify the surface of zirconia to improve the surface bioactivity and enhanced the osseointegration, such as physical, chemical, and pharmacologic modifications of the surface ${ }^{13,14)}$. In previous animal experiments, a rough surface of the zirconia material resulted in better osseointegration ${ }^{15-17)}$. However, in a review article comparing zirconia implants and titanium implants in animal models ${ }^{8)}$, most researchers reported that there was no significant difference between the two kinds of implants in osseointegration, while others reported a lower osseointegration ratio in zirconia implants. Therefore, it is still necessary to improve the surface bioactivity of zirconia materials.

Bioactive components on an implant surface can enhance initial osseointegration, which ensures successful implantation, especially for patients with periodontitis or decreased bone volume ${ }^{18}$. When adding bioactive components to an implant surface, a delivery carrier is required. Silk fibroin is a naturally occurring protein polymer and is widely used in tissue engineering fields because of its excellent biocompatibility ${ }^{19}$. With good mechanical properties and slow degradation profiles, it can be used as a drug delivery carrier ${ }^{20,21}$. Silk fibroin coatings have also been introduced to the dental field and are usually used to modify the surface of implants to obtain better osseointegration ${ }^{22,23)}$. In our previous research, silk fibroin was cationized with 
tetracycline, leading to a cationized antibacterial coating on the surface of titanium ${ }^{24}$. Electrophoretic deposition is usually employed to form a silk fibroin coating on titanium implants. However, according to some reports, owing to the relatively low mobility of oxygen vacancies, the conductivity of zirconia is low at normal temperatures $\left(<800^{\circ} \mathrm{C}\right)$ and it is not possible to form electrogel coatings directly on the surface of zirconia substrates ${ }^{25,26)}$. Therefore, it is necessary to cover a zirconia substrate with an electrogel after it forms in solution. Electrogels made from a high concentration silk fibroin solution are too dense to form uniform and smooth coatings on a substrate; therefore, a low concentration silk fibroin electrogel was applied in this study. This is the first study to introduce a silk fibroin electrogel for surface modification of zirconia.

The purpose of this study was to investigate the effect of a silk fibroin electrogel coating on the surface properties and biocompatibilities of Y-TZP and NANOZR compared to those of pure titanium (CpTi) to determine the feasibility of using silk fibroin as a drug delivery carrier for zirconia implants. In addition, we measured the bond strength between the silk fibroin electrogel and zirconia materials to calculate the coating effect from a mechanical point of view.

\section{MATERIALS AND METHODS}

\section{Specimen preparation}

Disks $15 \mathrm{~mm}$ in diameter and $1.5 \mathrm{~mm}$ in thickness made of $3 \mathrm{~mol} \%$ yttria-stabilized zirconia (3Y-TZP) (GC, Tokyo, Japan) and ceria-stabilized zirconia/ alumina nanocomposite (NANOZR; Panasonic Health Care, Tokyo, Japan) were used as substrates. The same shape of CpTi (Nippon Steel, Tokyo, Japan) was used as the control group. All specimens were polished with aluminum oxide waterproof abrasive paper (180\#, 400\#, and 600\#) and then grit-blasted perpendicularly from a distance of $10 \mathrm{~mm}$ with $125-\mu \mathrm{m}$ alumina particles at 0.4 $\mathrm{MPa}$ air pressure until the surface reached a uniform roughness. Then the ceramic specimens were etched in 47\% hydrofluoric acid (HF) for 15 min and the titanium specimens were etched in hydrochloric acid/sulfuric acid (1:1) at $70^{\circ} \mathrm{C}$ for $3 \mathrm{~min}$. These sand-blasted and acidetched (SLA) specimens were cleaned ultrasonically in acetone, ethanol and ultrapure water for $30 \mathrm{~min}$ and used as a control group ${ }^{27)}$.

The silk fibroin aqueous solution ( $\mathrm{pH}$ 6.4) (pH meter: SK-620PH, SATO KEIRYOKI MFG., Tokyo, Japan) was prepared according to standard protocol ${ }^{28)}$. According to the protocol, the residual salt in the solution was reduced to less than $10^{-11}$ of its original concentration, which can be considered to have no impact on the results. Then the solution was stored at $4^{\circ} \mathrm{C}$ for two weeks. The molecular weight of the silk fibroin solution prepared with the same protocol was reported to be $50-200 \mathrm{kDa}^{29)}$. Then, the silk fibroin solution was added into the electrophoresis tank and diluted to $30 \mathrm{mg} / \mathrm{mL}$. A platinum plate anode and a titanium plate cathode were placed into the tank at a distance of $8 \mathrm{~mm}$ from each other, and then, a direct current power supply was connected to the cathode and the anode. After applying a constant voltage of $25 \mathrm{~V}$ for $1.5 \mathrm{~h}$, a nontransparent aggregate of silk fibroin electrogel (SF) formed around the anodic platinum plate.

The SF was sucked out very gently and slowly with a pipette tip with a diameter larger than $5 \mathrm{~mm}^{30}$. The SLA specimens of CpTi, 3Y-TZP and NANOZR were covered with approximately $500 \mu \mathrm{L}$ electrogel and then air-dried for $48 \mathrm{~h}$.

To determine the electrogelation time, the relative density was detected in this study. A $25 \mathrm{~V}$ direct current was applied for $0.5,1,1.5$ and $2 \mathrm{~h}$, the electrogel from different distances away from the anode was then moved into a 96 -well plate. The density of the electrogel decreased as the distance from the anode increased. The optical density was detected using BIO-RAD iMark Microplate Reader (Bio-Rad, Hercules, CA, USA).

\section{Analyses of surface properties}

The coating effect was examined with attenuated total reflection Fourier transform infrared spectroscopy (ATR-FTIR; FT/IR 6300, JASCO, Tokyo, Japan). The silk fibroin electrogel-coated specimens and silk fibroin solution-coated specimens, which were completely air dried after the coating process were set on a single-reflection diamond ATR attachment. Spectral wavenumbers between 1,100-1,800 $\mathrm{cm}^{-1}$ were observed with analysis software (JASCO).

The surface wettability was analyzed using contact angle measurements. The contact angle was examined with a portable contact angle meter (PCA-1, Kyowa Interface Science, Saitama, Japan). Drops of Milli-Q water with a volume of $2 \mu \mathrm{L}$ were placed on a flat material surface with a micrometric syringe. The examination was carried out at room temperature $\left(23 \pm 2^{\circ} \mathrm{C}\right)$ with a humidity of $50 \%$. Images were analyzed with FAMAS software (Kyowa Interface Science). Ten measurements were obtained with 5 specimens of each group.

The shear bond strength and tensile bond strength were measured to analyze the coating adhesion. Two SFcoated specimens were stacked together with the wetcoating surfaces facing each other to create a detection unit. After the units were thoroughly air dried, the bond strength was measured using a material testing machine (Model 5565, Instron, Norwood, MA, USA) in shear mode and tensile mode with a crosshead speed of $0.5 \mathrm{~mm} / \mathrm{min}$ at room temperature $\left(23 \pm 2^{\circ} \mathrm{C}\right)$ until complete separation occurred. Five samples per group were used. The modes of debonding were classified as cohesive (delamination inside the coating) and adhesive (delamination from the coating-substrate interface). Photoshop CS 6 was used to measure the area of cohesive and adhesive parts to determine the adhesive/cohesive ratio.

\section{Cell morphologies and cell proliferation on the surface} of specimens

In this study, MC3T3-E1 osteoblast-like cells were used to evaluate the cell morphology and proliferation on SFcoated surfaces of CpTi, 3Y-TZP and NANOZR. Cells were cultured in Dulbecco's modified eagle's medium 
(DMEM; Nacalai Tesque, Kyoto, Japan) with 10\% fetal bovine serum (FBS; Gibco, New York, NY, USA).

MC3T3-E1 cell suspensions with a cell density of $4 \times 10^{3}$ cells $/ \mathrm{mL}$ were added to each well of a 24 -well plate. After being cultured for 2 days at $37^{\circ} \mathrm{C}$ in a humidified $5 \% \mathrm{CO}_{2}$ incubator, the specimens were rinsed twice with phosphate-buffered saline solution (PBS; $\mathrm{pH}$ 7.2) to remove unattached cells and then fixed with $2.5 \%$ glutaraldehyde solution (Sigma, St. Louis, MO, USA) for 1 day. Then the fixed cells were dehydrated progressively in a graded series of ethanol $(50,60,70,80,90,95$ and $100 \%$ ) for $15 \mathrm{~min}$. The dehydrated samples were gold sputter-coated and examined by SEM (JSM 6390LA, JEOL, Tokyo, Japan). For cell skeleton and nucleus observation, the attached cells were fixed with $4 \%$ paraformaldehyde solution (Sigma) for 1 day and permeabilized with $0.1 \%$ Triton-X100 (Amresco, Cleveland, OH, USA) for 5 min at room temperature. After incubating the samples in PBS containing 1\% bovine serum albumin for 30 min to block nonspecific binding sites, cells were stained with rhodamine phalloidin (Cytoskeleton, Denver, CO, USA) at room temperature for $30 \mathrm{~min}$ and then finally stained with DAPI (Invitrogen, Basel, Switzerland) for $30 \mathrm{~s}$. Immunofluorescence images were obtained using a laser scanning confocal microscope (TCS SP5, Leica, Tokyo, Japan) ${ }^{12}$.

MC3T3-E1 cells with a concentration of $2 \times 10^{4}$ cells/ $\mathrm{mL}$ were seeded in a 24 -well culture plate with six kinds of specimens inside. The quantity of attached cells was determined using cell counting kit-8 (CCK- 8, Dojindo, Kumamoto, Japan) after cell seeding for 1, 3, 5 and 7 days. After $10 \mu \mathrm{L}$ of CCK-8 solution was added into each well containing $100 \mu \mathrm{L}$ of cell culture medium and incubated for $2 \mathrm{~h}$, the absorbance was measured at 450 nm using a BIO-RAD iMark Microplate Reader (BioRad). All experiments were performed three times.

\section{Statistical analysis}

All data were analyzed independently by one-way analysis of variance (ANOVA) combined with Tukey's multiple comparison test at a $5 \%$ level of significance.

\section{RESULTS}

\section{Surface properties of specimens}

As the electrophoresis time elapsed, a silk gel formed on the anode. The thick gel leads to a decay in the actual current. Figure 1 shows that after $25 \mathrm{~V}$ of direct current was applied, the density of the electrogel increased over time. However, at $2 \mathrm{~h}$, the electrogel coating on the anode grew too thick to maintain the efficiency of electrogelation, therefore, a time of $1.5 \mathrm{~h}$ was applied, because the electrogelation became less efficient after that time.

The ATR-FTIR spectra are shown in Fig. 2. The changes in the characteristic peak after electrogelation can be seen in these spectra. In specimens coated by the silk fibroin solution, the characteristic peaks were observed at approximately 1,640, 1,518 and 1,236

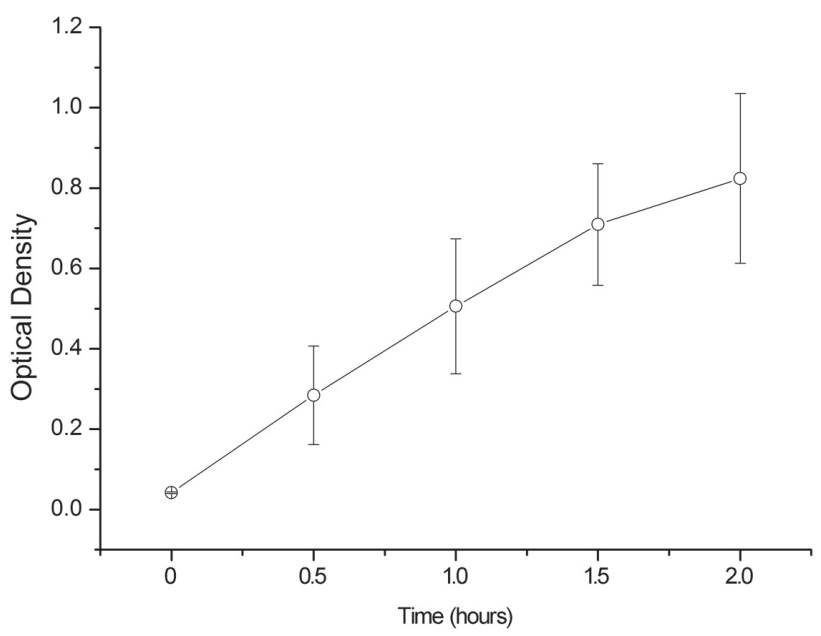

Fig. 1 Density of the electrogel at different electrophoresis times.

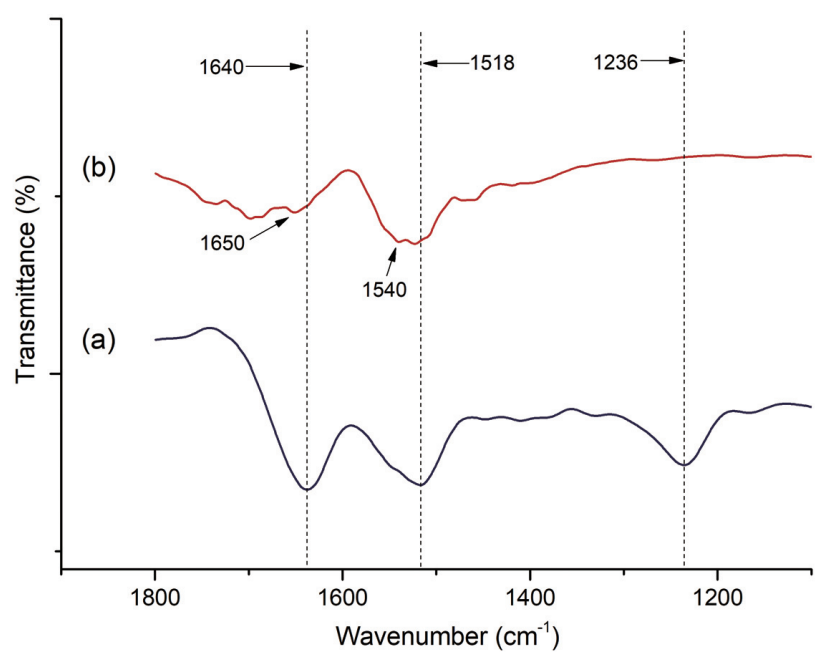

Fig. 2 ATR-FTIR spectra of coatings, where wavenumbers between $1,100-1,800 \mathrm{~cm}^{-1}$ were observed.

The characteristic peaks for amide I at $1,640 \mathrm{~cm}^{-1}$ and amide II at $1,518 \mathrm{~cm}^{-1}$ were observed for the (a) silk fibroin solution. The characteristic peaks for amide I was at $1,650 \mathrm{~cm}^{-1}$ and amide II was at $1,540 \mathrm{~cm}^{-1}$ in (b) silk fibroin electrogels.

$\mathrm{cm}^{-1}$, while for the specimens coated by the silk fibroin electrogel, the characteristic peaks were observed at approximately 1,650 and $1,540 \mathrm{~cm}^{-1}$.

Figure 3 shows the contact angles measured under static conditions. For the zirconia materials, there was no significant difference between the SLA specimens and SF coated specimens $(p>0.05)$, while for $\mathrm{CpTi}$, the contact angle significantly decreased after electrogelcoated $(p<0.05)$.

The results of the shear bond strength and the tensile bond strength are shown in Fig. 4. No significant difference was obsearevd in the shear bond strength and 


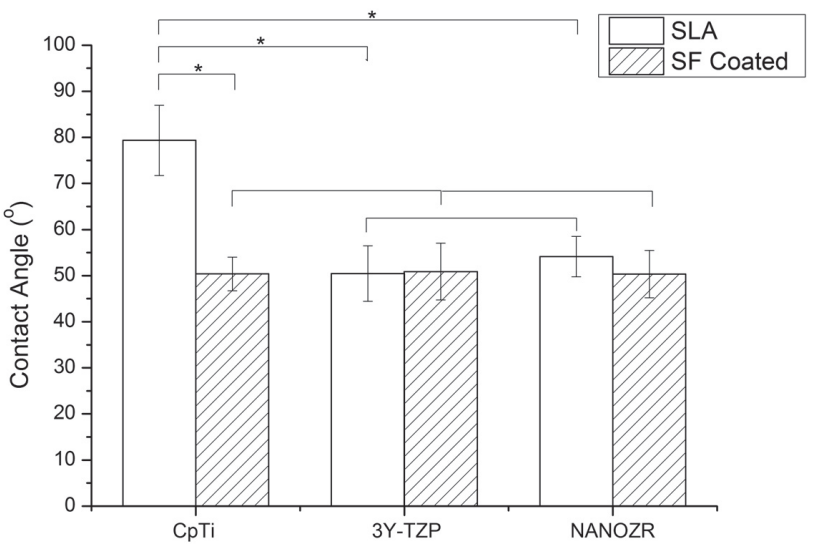

Fig. 3 Water contact angle of SLA and electrogel-coated surfaces.

The contact angle on the SLA CpTi surface was statistically larger than that of the other samples $\left({ }^{*} p \leq 0.05\right)$.
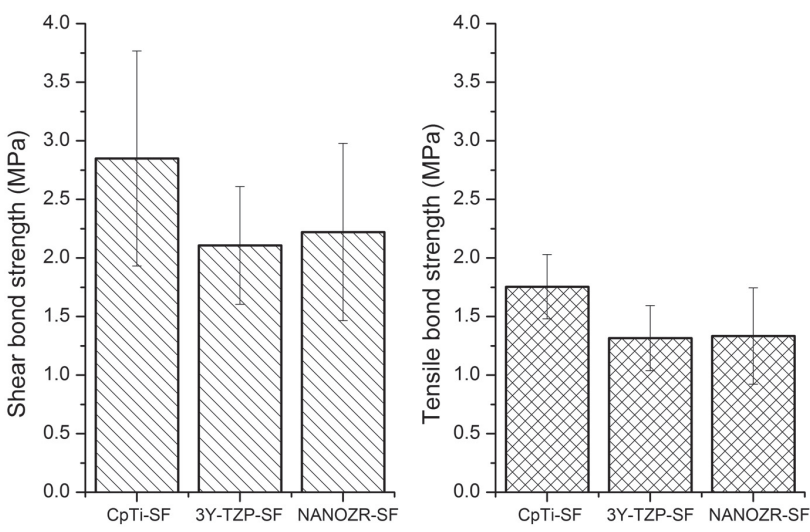

Fig. 4 Shear bond strength and tensile bond strength of electrogel coatings on CpTi, 3Y-TZP and NANOZR substrates.
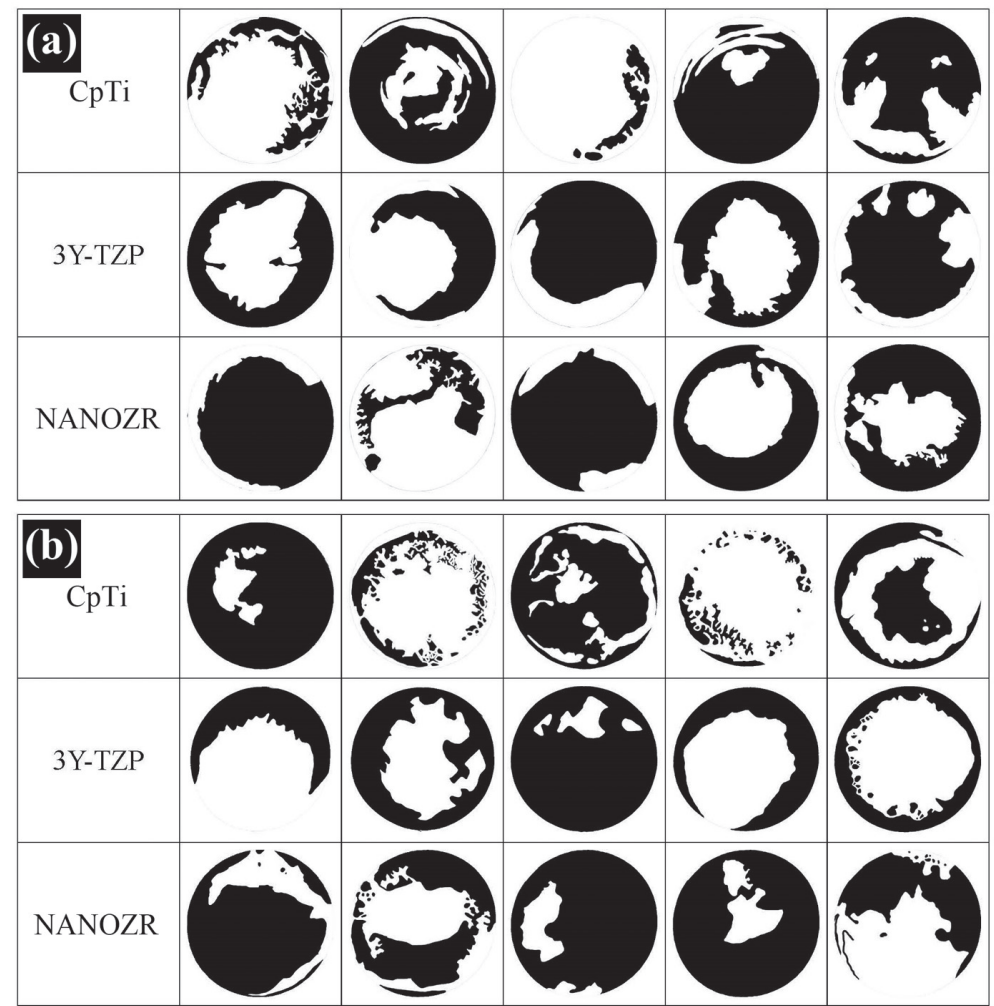

Fig. 5 Fragmentation patterns after shear bond strength test (a) and tensile bond strength test (b).

The white color area presents separation from the interface, and the black color area presents delamination inside the coating. Typical images are shown.

tensile bond strength among the three kinds of materials after $\mathrm{SF}$ coated $(p>0.05)$. Images of the debonding mode at the interface between the silk fibroin electrogel and specimens are shown in Fig. 5. The percentages of adhesive failure were 52.23, 55.15, and 39.91\% for $\mathrm{CpTi}$,
3Y-TZP and NANOZR, respectively in shear mode and $56.67,61.72$, and $38.56 \%$ for CpTi, 3Y-TZP and NANOZR, respectively in tensile mode. 

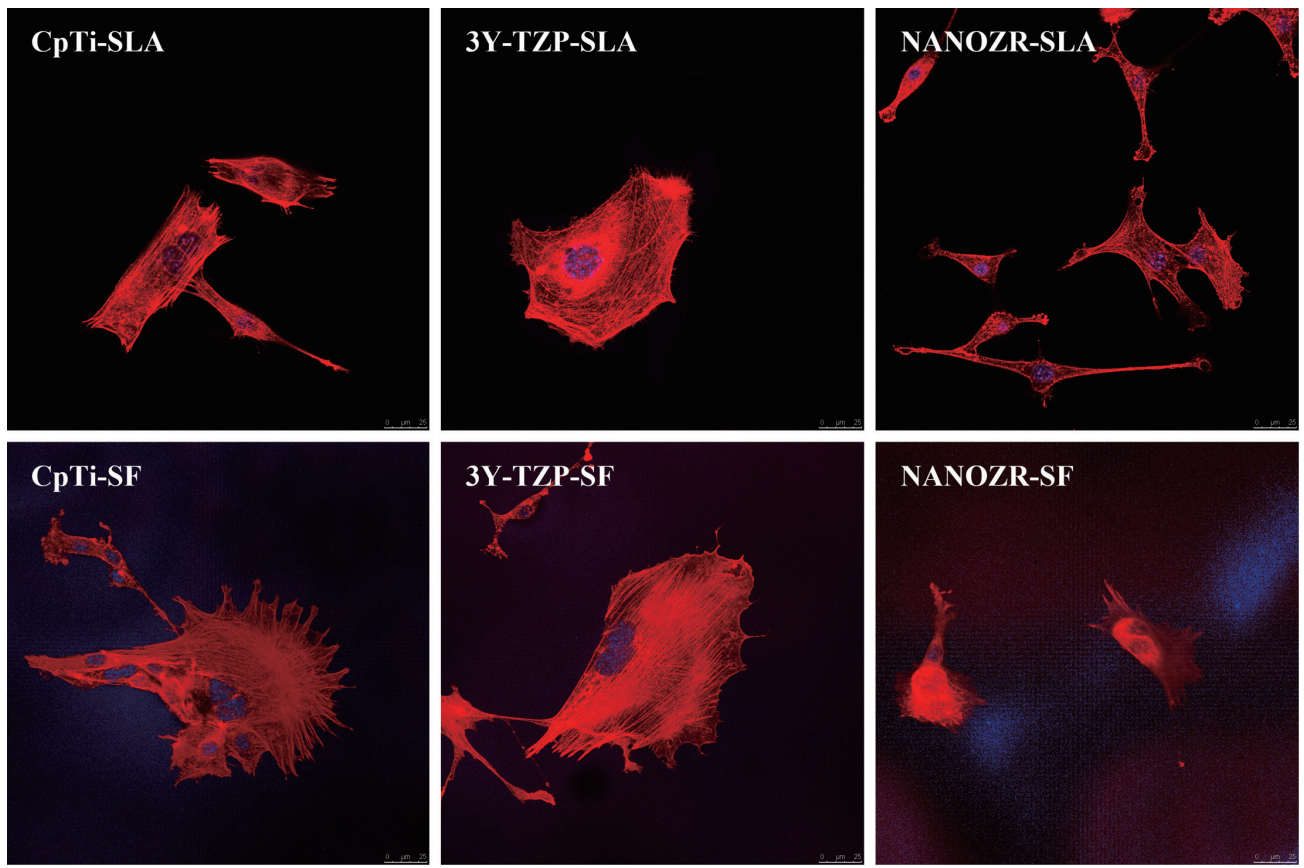

Fig. 6 Fluorescence microscopy images of MC3T3-E1 cells on SLA and electrogel-coated surfaces after two days of culture.

Microphotographs are of typical fields.
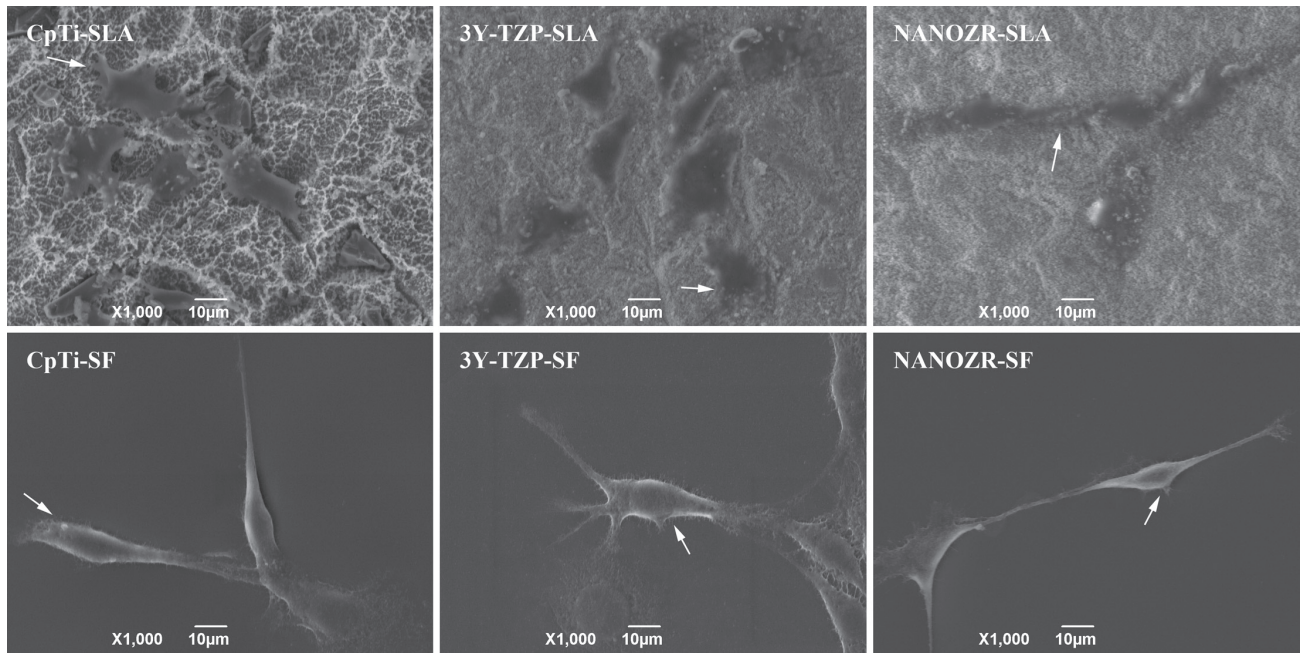

Fig. 7 SEM images of MC3T3-E1 cells on SLA and electro-gel-coated surfaces after two days of culture.

Numerous filopodia can be observed (white arrow). Microphotographs are of typical fields.

Cell morphologies and cell proliferation on the surface of the specimens

Immunofluorescence images of the actin cytoskeleton and SEM images of the cell morphology are shown in Figs. 6 and 7. After 2 days of incubation, MC3T3-E1 cells appeared to attach and spread well on both the SLAand SF-coated surfaces of the three kinds of materials. Cell-cell contacts and filopodia were observed clearly in all specimens. For the cell morphology, there was no obvious difference between the SLA surfaces and SFcoated surfaces.

The results of proliferation of MC3T3-E1 cells is shown in Fig. 8. The cell number significantly increased during the 7-day test period. No significant difference was shown among the different specimens after being cultured for 7 days. 


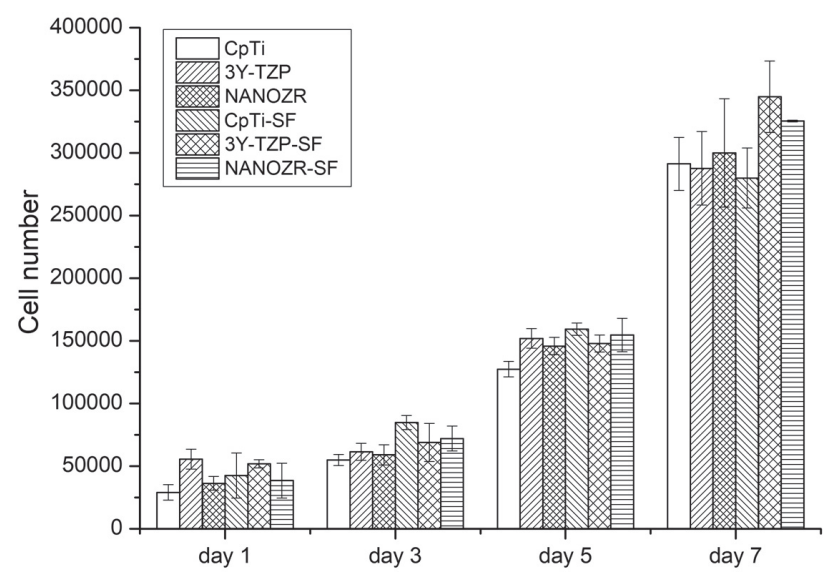

Fig. 8 The proliferation of MC3T3-E1 cells after culturing for 1, 3, 5 and 7 days on SLA and electrogel-coated surfaces examined using a CCK-8 kit.

There was no significant difference among the six groups within the tested period $(p>0.05)$.

\section{DISCUSSION}

Silk fibroin has been applied in the biomedical field for many years and has mainly been processed into films, porous scaffolds, microspheres and hydrogels ${ }^{31)}$. In its fresh aqueous solution, silk fibroin exists as a randomcoil-rich conformation. The ratio of $\alpha$-helix and $\beta$-sheet in a silk fibroin solution varies with different conditions, such as temperature, storage time, $\mathrm{pH}$ value, additional direct current and mechanical force. The structural changes of a silk fibroin solution tend to result in reversible or irreversible aggregation ${ }^{32,33)}$. In this study, a direct current was applied to prepare a silk fibroin electrogel.

To further explore the microstructure of the electrogel, the surface of specimens coated by the electrogel was characterized by ATR-FTIR spectra. In the range of $1,000-1,800 \mathrm{~cm}^{-1}$, secondary structural changes were observed between the silk fibroin solution and the silk fibroin electrogel. After the electrogelation process, the amide I peak shifted from 1,640 to 1,650 $\mathrm{cm}^{-1}$ and the amide II peak shifted from 1,518 to 1,540 $\mathrm{cm}^{-1}$, which could be attributed to the increase in the $\alpha$-helix content. In the spectrum of the electrogel, no peak or shoulder appeared at approximately $1,236 \mathrm{~cm}^{-1}$, suggesting a decrease or disappearance of random coils. The results agreed with the quantitative experiments conducted by Lu et al., they reported that in a fresh silk fibroin solution, the ratios of random coil and $\alpha$-helix are $26.1 \pm 2.6$ and $11.0 \pm 1.5$, respectively, while in the electrogel, the corresponding ratios are $15.7 \pm 1.7$ and $16.2 \pm 2.0$, respectively ${ }^{33)}$.

It is well known that tissue engineering starts from cell seeding on an artificial substrate. A proper substrate surface will promote cell adhesion, migration, proliferation and differentiation. An inverse relationship was found between the contact angle and blood clot
Table 1 The contact angle of specimens' surface

\begin{tabular}{lccc}
\hline & CpTi & 3Y-TZP & NANOZR \\
\hline SLA & $79.35 \pm 7.62$ & $50.46 \pm 6.01$ & $54.14 \pm 4.36$ \\
SF coated & $50.38 \pm 3.65$ & $50.88 \pm 6.16$ & $50.33 \pm 5.12$ \\
\hline
\end{tabular}

extension, while the latter plays an important role in the initial endothelial cell response for vessel repair ${ }^{34)}$. For dental implants, surface wettability is an important parameter affecting osseointegration. In this study, the contact angle on the surface of SLA treated CpTi was approximately $80^{\circ}$ (Table 1 ), which was significantly higher than that of the electrogel-coated specimens and zirconia materials, which was approximately $50^{\circ}$. However, for zirconia materials, there was no significant difference between the electrogel-coated and uncoated specimens. These results suggested that the electrogel coating increased the wettability of $\mathrm{CpTi}$, but had no significant impact on the zirconia specimens.

Surface treatment prior to the coating process is also a concern. Silk fibroin is a biodegradable material, which means that the bone contact of the substrate after degradation is also important. Gahlert et al. reported that roughened surfaces improved early cell attachment to titanium ${ }^{35)}$; similarly, Yamashita et al. found that roughened zirconia surfaces had a beneficial effect on cell attachment and initial cell adhesion ${ }^{11)}$. To obtain a rougher surface, sandblast and acid etching are commonly used methods. According to results reported in vivo, the combination of acid etching and sandblasting can accelerate bone regeneration ${ }^{36)}$. Elia et al. ${ }^{37)}$ reported that a higher adhesive strength was obtained on a rougher surface when the same concentration of silk fibroin was applied for a coating. Therefore, SLA substrates were used for surface coating by an electrogel in this study. The adhesive strength of the hydrate silk coating on titanium substrates was tested, and the results indicated that the adhesive strength was in a range from $0.1-0.88 \mathrm{MPa}$ and depended on the concentration of the silk fibroin solution and the substrate surface treatment ${ }^{30)}$. In this study, the bonding strength of the electrogel coating was higher than that obtained with the hydrate silk coating. This result was assumed to be mainly due to the eletrogelation process, which is briefly summarized as follows. In a neutral aqueous solution, the negative surface charge of silk fibroin prevents intermolecular self-assembly. When a direct current is added to an anode and cathode in solution, the proton concentration near the anode increases, which results in a local decrease in the $\mathrm{pH}$ value. Because of the acidic surface charged groups, the balance of the electrical charge is broken and silk fibroin can intermolecularly self-assemble, forming nanospheres and microsphere ${ }^{31)}$. To further understand the adhesion at the coatingsubstrate interface, fragmentation patterns were analyzed. The ratio of cohesive failure and adhesive failure was always used to analyze the interface 
performance of adhesion behavior. The former controls delamination inside a coating, while the latter controls failure at the coating-substrate interface ${ }^{38}$. For the NANOZR substrate, the ratio of adhesive failure was lower than that of the others in both the shear bond test and tensile bond test, revealing that more fragmentation occurred inside the coating rather than at the interface. This result suggested that the adhesion of the electrogel to NANOZR may be stronger than the adhesion to $\mathrm{CpTi}$ and 3Y-TZP. The results may also indicate a sufficient bonding strength for clinical use when the electrogel coating method is used to implant materials.

Silk is well-known as a biocompatible material because of the long history of clinical use of silk sutures. In recent years, some silk-based biomaterials have achieved success when applied in plastic and reconstructive surgery ${ }^{39}$. The biocompatibility of electrogel coating on titanium surfaces has also been reported ${ }^{24)}$. However, when coated on different substrates, the ions in the coating may change, and this change may affect the biocompatibility of materials coated by a silk fibroin electrogel. In the present study, the cell skeleton, morphology and proliferation were observed to confirm whether the coating is an optimal surface for cells. The cytoskeleton, which plays an important role in structural framework and cell migration ${ }^{40)}$, was clearly observed in the immunofluorescence image. The cells spread widely and anchored to the surface through long filopodia on all six kinds of surfaces after $48 \mathrm{~h}$ culture. Moreover, SEM images showed slender filopodia crossed to link with adjacent cells, which could mediate intercellular transfer of organelles and proteins and thus promote cell-to-cell communication. The CCK- 8 data showed that the cell numbers were comparable on the six kinds of surfaces on day 1, which means that the SLA specimens were as favorable as the electrogel-coated specimens for the initial attachment of MC3T3 cells. The long-term culture showed a similar tendency of increasing cell number, with no significant influence of the silk fibroin coating on cell proliferation.

The silk fibroin biodegradation was also studied in vitro and in vivo. In report from Wang et al. based on implantation in rats $^{41}$, the degradation time for scaffolds prepared from an all-aqueous process was $2-6$ months. The time for the completion of degradation mainly depends on the silk fibroin concentration and pore size inside the scaffolds. In Elia's study ${ }^{37)}$, researchers explored drug release from an electrodeposited silk fibroin coating using FITC-dextrans. The drug release curve was in line with that observed in traditional systems. Other small molecule drugs such as antibiotics were also successfully released from an electrogel according to their unpublished data. Therefore, it is possible to make a drug-delivery system on the surface of a zirconia implant material using silk fibroin electrogel coating.

This study was not able to accurately simulate the clinical conditions, and biocompatibility was examined in vitro. Additionally, the bioactivity of materials is strongly affected by the experimental conditions. Therefore, an in vivo study involving animal experiments is necessary to overcome the limitations of the in vitro study. The performance of a silk fibroin electrogel coating on zirconia implants still warrants further investigation.

\section{CONCLUSION}

Within the limitations of this study, the results suggested that a silk fibroin electrogel coating has sufficient bonding strength and may be useful in improving the biocompatibility of zirconia materials. The silk fibroin electrogel could be a promising drug-delivery system for zirconia implant materials.

\section{ACKNOWLEDGMENTS}

This research was supported by the Translational Research Network Program Seeds A (No. A-72) for Scientific Research from the Ministry of Education, Culture, Sports, Science and Technology, Japan and the Natural Science Foundation of Liaoning Province of China (No. 20180550538).

\section{REFERENCES}

1) Delaunay C. Twenty-year survival of a grit-blasted titanium threaded cup in primary total hip arthroplasty: The swan song. Orthop Proc 2018; 100-B: 55-55.

2) Elias CN, Lima JHC, Valiev R, Meyers MA. Biomedical applications of titanium and its alloys. JOM 2008; 60: 46-49.

3) Liu L, Qu YY, Jiang LJ, Zhou Q, Tang TQ. Three-dimensional finite element analysis of a newly designed onplant miniplate anchorage system. J Huazhong Univ Sci Technolog Med Sci 2016; 36: 422-427.

4) Meyer U, Buhner M, Buchter A, Kruse-Losler B, Stamm T, Wiesmann HP. Fast element mapping of titanium wear around implants of different surface structures. Clin Oral Implants Res 2006; 17: 206-211.

5) Weingart D, Steinemann S, Schilli W, Strub JR, Hellerich $\mathrm{U}$, Assenmacher J, et al. Titanium deposition in regional lymph nodes after insertion of titanium screw implants in maxillofacial region. Int J Oral Surg 1994; 23: 450-452.

6) Depprich R, Zipprich H, Ommerborn M, Naujoks C, Wiesmann HP, Kiattavorncharoen S, et al. Osseointegration of zirconia implants compared with titanium: an in vivo study. Head Face Med 2008; 4: 30 doi: 10.1186/1746-160X-4-30.

7) Ozkurt Z, Kazazoglu E. Zirconia dental implants: a literature review. J Oral Implantol 2011; 37: 367-376.

8) Manzano G, Herrero LR, Montero J. Comparison of clinical performance of zirconia implants and titanium implants in animal models: a systematic review. Int $\mathrm{J}$ Oral Maxillofac Implants 2014; 29: 311-320.

9) Chevalier J. What future for zirconia as a biomaterial? Biomaterials 2006; 27: 535-543.

10) Nawa $M$, Nakamoto $S$, Sekino $T$, Niihara $K$. Tough and strong Ce-TZP/Alumina nanocomposites doped with titania. Ceram Int 1998; 24: 497-506.

11) Yamashita D, Machigashira M, Miyamoto M, Takeuchi $\mathrm{H}$, Noguchi K, Izumi Y, et al. Effect of surface roughness on initial responses of osteoblast-like cells on two types of zirconia. Dent Mater J 2009; 28: 461-470.

12) Han JM, Hong G, Matsui H, Shimizu Y, Zheng G, Lin H, et $a l$. The surface characterization and bioactivity of NANOZR in vitro. Dent Mater J 2014; 33: 210-219.

13) Langhoff JD, Voelter K, Scharnweber D, Schnabelrauch M, Schlottig F, Hefti T, et al. Comparison of chemically and pharmaceutically modified titanium and zirconia implant 
surfaces in dentistry: a study in sheep. Int J Oral Surg 2008; 37: 1125-1132

14) Liu J, Hong G, Wu YH, Endo K, Han JM, Kumamoto H, et $a l$. A novel method of surface modification by electrochemical deoxidation: Effect on surface characteristics and initial bioactivity of zirconia. J Biomed Mater Res B Appl Biomater 2017; 105: 2641-2652.

15) Rocchietta I, Fontana F, Addis A, Schupbach P, Simion M. Surface-modified zirconia implants: tissue response in rabbits. Clin Oral Implants Res 2009; 20: 844-850.

16) Kohal RJ, Wolkewitz M, Hinze M, Han JS, Bachle M, Butz F. Biomechanical and histological behavior of zirconia implants: an experiment in the rat. Clin Oral Implants Res 2009; 20: 333-339.

17) Koch FP, Weng D, Kramer S, Biesterfeld S, Jahn-Eimermacher A, Wagner W. Osseointegration of one-piece zirconia implants compared with a titanium implant of identical design: a histomorphometric study in the dog. Clin Oral Implants Res 2010; 21: 350-356.

18) Yoo D, Tovar N, Jimbo R, Marin C, Anchieta RB, Machado LS, et al. Increased osseointegration effect of bone morphogenetic protein 2 on dental implants: an in vivo study. J Biomed Mater Res A 2014; 102: 1921-1927.

19) Kasoju N, Bora U. Silk fibroin in tissue engineering. Adv Healthcare Mater 2012; 1: 393-412.

20) Mottaghitalab F, Farokhi M, Shokrgozar MA, Atyabi F, Hosseinkhani H. Silk fibroin nanoparticle as a novel drug delivery system. J Control Release 2015; 206: 161-176.

21) Zhao Z, Li Y, Xie MB. Silk fibroin-based nanoparticles for drug delivery. Int J Mol Sci 2015; 16: 4880-4903.

22) Elia R, Michelson CD, Perera AL, Harsono M, Leisk GG, Kugel G, et al. Silk electrogel coatings for titanium dental implants. J Biomater Appl 2015; 29: 1247-1255.

23) Zhang Z, Jiang T, Ma K, Cai X, Zhou Y, Wang Y. Low temperature electrophoretic deposition of porous chitosan/silk fibroin composite coating for titanium biofunctionalization. J Mater Chem 2011; 21: 7705-7713.

24) Zhang $\mathrm{Z}, \mathrm{Qu} \mathrm{Y}, \mathrm{Li} \mathrm{X}$, Zhang S, Wei Q, Shi Y, et al. Electrophoretic deposition of tetracycline modified silk fibroin coatings for functionalization of titanium surfaces. Appl Surf Sci 2014; 303: 255-262.

25) Guo X. Can we achieve significantly higher ionic conductivity in nanostructured zirconia? Scr Mater 2011; 65: 96-101.

26) Kosacki I, Suzuki T, Petrovsky V, Anderson HU. Electrical conductivity of nanocrystalline ceria and zirconia thin films. Solid State Ionics 2000; 136-137: 1225-1233.

27) Takano T, Tasaka A, Yoshinari M, Sakurai K. Fatigue strength of Ce-TZP/Al2O3 nanocomposite with different surfaces. J Dent Res 2012; 91: 800-804.
28) Rockwood DN, Preda RC, Yucel T, Wang X, Lovett ML, Kaplan DL. Materials fabrication from Bombyx mori silk fibroin. Nat Protoc 2011; 6: 1612-1631.

29) Wang HY, Zhang YQ. Effect of regeneration of liquid silk fibroin on its structure and characterization. Soft Matter 2013; 9: 138-145.

30) Leisk GG, Lo TJ, Yucel T, Lu Q, Kaplan DL. Electrogelation for protein adhesives. Adv Mater 2010; 22: 711-715.

31) Yucel T, Kojic N, Leisk GG, Lo TJ, Kaplan DL. Nonequilibrium silk fibroin adhesives. J Struct Biol 2010; 170: 406-412.

32) Matsumoto A, Chen J, Collette AL, Kim UJ, Altman GH, Cebe $\mathrm{P}$, et al. Mechanisms of silk fibroin sol-gel transitions. J Phys Chem B 2006; 110: 21630-21638.

33) Lu Q, Huang Y, Li M, Zuo B, Lu S, Wang J, et al. Silk fibroin electrogelation mechanisms. Acta Biomater 2011; 7: 23942400.

34) Traini T, Caputi S, Gherlone E, Degidi M, Piattelli A. Fibrin clot extension on zirconia surface for dental implants: a quantitative in vitro study. Clin Implant Dent Relat Res 2014; 16: 718-727.

35) Gahlert M, Gudehus T, Eichhorn S, Steinhauser E, Kniha H, Erhardt W. Biomechanical and histomorphometric comparison between zirconia implants with varying surface textures and a titanium implant in the maxilla of miniature pigs. Clin Oral Implants Res 2007; 18: 662-668.

36) Herrero-Climent M, Lazaro P, Vicente Rios J, Lluch S, Marques M, Guillem-Marti J, et al. Influence of acid-etching after grit-blasted on osseointegration of titanium dental implants: in vitro and in vivo studies. J Mater Sci Mater Med 2013; 24: 2047-2055

37) Elia R, Michelson CD, Perera AL, Brunner TF, Harsono M, Leisk GG, et al. Electrodeposited silk coatings for bone implants. J Biomed Mater Res B Appl Biomater 2015; 103: 1602-1609.

38) Leterrier Y, Andersons J, Pitton Y, Månson JAE. Adhesion of silicon oxide layers on poly(ethylene terephthalate). II: Effect of coating thickness on adhesive and cohesive strengths. J Polym Sci B Polym Phys 2015; 35: 1463-1472.

39) Kundu B, Rajkhowa R, Kundu SC, Wang X. Silk fibroin biomaterials for tissue regenerations. Adv Drug Deliv Rev 2013; 65: 457-470.

40) Salido M, Vilches JI, Gutierrez JL, Vilches J. Actin cytoskeletal organization in human osteoblasts grown on different dental titanium implant surfaces. Histol Histopathol 2007; 22: 13551364.

41) Wang Y, Rudym DD, Walsh A, Abrahamsen L, Kim HJ, Kim $\mathrm{HS}$, et al. In vivo degradation of three-dimensional silk fibroin scaffolds. Biomaterials 2008; 29: 3415-3428. 